\title{
On Some New Hermite-Hadamard Type Inequalities for $s$-Geometrically Convex Functions
}

\author{
İmdat İşcan \\ Department of Mathematics, Faculty of Arts and Sciences, Giresun University, 28200 Giresun, Turkey \\ Correspondence should be addressed to İmdat İşcan; imdat.iscan@giresun.edu.tr \\ Received 30 December 2013; Revised 7 June 2014; Accepted 9 June 2014; Published 19 June 2014 \\ Academic Editor: Henryk Hudzik
}

Copyright (c) 2014 İmdat İșcan. This is an open access article distributed under the Creative Commons Attribution License, which permits unrestricted use, distribution, and reproduction in any medium, provided the original work is properly cited.

Some new integral inequalities of Hermite-Hadamard type related to the $s$-geometrically convex functions are established and some applications to special means of positive real numbers are also given.

\section{Introduction}

In this section, we firstly list several definitions and some known results.

Definition 1. Let $I$ be an interval in $\mathbb{R}$. Then $f: I \rightarrow \mathbb{R}$ is said to be convex if

$$
f(\lambda x+(1-\lambda) y) \leq \lambda f(x)+(1-\lambda) f(y)
$$

for all $x, y \in I$ and $\lambda \in[0,1]$.

Definition 2 (see [1]). Let $s \in(0,1]$. A function $f: I \subset$ $[0, \infty) \rightarrow[0, \infty)$ is said to be $s$-convex in the second sense if

$$
f(\lambda x+(1-\lambda) y) \leq \lambda^{s} f(x)+(1-\lambda)^{s} f(y)
$$

for all $x, y \in I$ and $\lambda \in[0,1]$.

Definition 3 (see [2]). A function $f: I \subset \mathbb{R}_{+}=(0, \infty) \rightarrow \mathbb{R}_{+}$ is said to be a geometrically convex function if

$$
f\left(x^{\lambda} y^{1-\lambda}\right) \leq f(x)^{\lambda} f(y)^{1-\lambda}
$$

for $x, y \in I$ and $\lambda \in[0,1]$.

Definition 4 (see [2]). A function $f: I \subset \mathbb{R}_{+}=(0, \infty) \rightarrow \mathbb{R}_{+}$ is said to be an $s$-geometrically convex function if

$$
f\left(x^{\lambda} y^{1-\lambda}\right) \leq f(x)^{\lambda^{s}} f(y)^{(1-\lambda)^{s}}
$$

for some $s \in(0,1]$, where $x, y \in I$ and $\lambda \in[0,1]$.
Example 5. (i) $f(x)=e^{x}, x>0$, is $s$-geometrically convex for all $0<s \leq 1$.

(ii) $f(x)=c, x>0$, is $s$-geometrically convex for all $0<$ $s \leq 1$, where $c \geq 1$ is a fixed number.

Let $f: I \subset \mathbb{R}_{+}=(0, \infty) \rightarrow[1, \infty)$. It is easy to show that $f$ is $s$-geometrically convex function on $[a, b], a, b \in I$ with $a<b$ if and only if $\ln (f \circ \exp )$ is $s$-convex function on $[\ln a, \ln b]$.

Let $f: I \subseteq \mathbb{R} \rightarrow \mathbb{R}$ be a convex function defined on the interval $I$ of real numbers and $a, b \in I$ with $a<b$. The following double inequality is well known in the literature as Hermite-Hadamard integral inequality:

$$
f\left(\frac{a+b}{2}\right) \leq \frac{1}{b-a} \int_{a}^{b} f(x) d x \leq \frac{f(a)+f(b)}{2} .
$$

Recently, several integral inequalities connected with inequalities (5) for the $s$-convex functions have been established by many authors; for example, see [1, 3-6]. In [2], the authors have established some integral inequalities connected with inequalities (5) for the $s$-geometrically convex and monotonically decreasing functions. In [7], Tunç has established inequalities for $s$-geometrically and geometrically convex functions which are connected with the famous Hermite-Hadamard inequality holding for convex functions. In [7], Tunç also has given the following result for geometrically convex and monotonically decreasing functions. 
Corollary 6. Let $f: I \subset \mathbb{R}_{+} \rightarrow \mathbb{R}_{+}$be geometrically convex and monotonically decreasing on $[a, b], a, b \in I$ with $a<b$; then one has

$$
f^{2}(\sqrt{a b}) \leq \frac{1}{\ln b-\ln a} \int_{a}^{b} \frac{f(x)}{x} f\left(\frac{a b}{x}\right) d x \leq f(a) f(b) .
$$

Note that inequalities (6) are also true without the condition monotonically decreasing and inequalities (6) are sharp. Also, inequality (6) obtained for geometrically convex functions is analogous with Hermite-Hadamard inequality (5).

Since $f: I \subset \mathbb{R}_{+} \rightarrow \mathbb{R}_{+}$is a geometrically convex function if and only if $\sqrt{f}$ is a geometrically convex function, inequality (6) can be given as follows:

$$
\begin{aligned}
f(\sqrt{a b}) & \leq \frac{1}{\ln b-\ln a} \int_{a}^{b} \frac{1}{x} \sqrt{f(x) f\left(\frac{a b}{x}\right)} d x \\
& \leq \sqrt{f(a) f(b)} .
\end{aligned}
$$

In this paper, the author gives new identities for differentiable functions. A consequence of the identities is that the author establishes some new inequalities connected with inequalities (6) for the $s$-geometrically convex functions.

\section{Main Results}

In order to prove our results, we need the following lemma.

Lemma 7. Let $f: I \subseteq \mathbb{R}_{+} \rightarrow \mathbb{R}$ be differentiable on $I^{\circ}$ and $a, b \in I$ with $a<b$. If $f^{\prime} \in L[a, b]$, then

$$
\begin{aligned}
f(a) f(b) & -\frac{1}{\ln b-\ln a} \int_{a}^{b} \frac{f(x)}{x} f\left(\frac{a b}{x}\right) d x \\
=\int_{0}^{1} & \frac{b}{2} \ln \left(\frac{a}{b}\right)(t-1)\left(\frac{a}{b}\right)^{t / 2} \\
& \times f\left(a^{1-t}(a b)^{t / 2}\right) f^{\prime}\left(b^{1-t}(a b)^{t / 2}\right) \\
& +\frac{a}{2} \ln \left(\frac{b}{a}\right)(t-1)\left(\frac{b}{a}\right)^{t / 2} \\
& \times f^{\prime}\left(a^{1-t}(a b)^{t / 2}\right) f\left(b^{1-t}(a b)^{t / 2}\right) d t, \\
f^{2}(\sqrt{a b}) & -\frac{1}{\ln b-\ln a} \int_{a}^{b} \frac{f(x)}{x} f\left(\frac{a b}{x}\right) d x \\
=\int_{0}^{1} & \frac{b}{2} \ln \left(\frac{a}{b}\right) t\left(\frac{a}{b}\right)^{t / 2} \\
& \times f\left(a^{1-t}(a b)^{t / 2}\right) f^{\prime}\left(b^{1-t}(a b)^{t / 2}\right) \\
& +\frac{a}{2} \ln \left(\frac{b}{a}\right) t\left(\frac{b}{a}\right)^{t / 2} \\
& \times f^{\prime}\left(a^{1-t}(a b)^{t / 2}\right) f\left(b^{1-t}(a b)^{t / 2}\right) d t .
\end{aligned}
$$

Proof. Integrating by part and changing variables of integration yield

$$
\begin{aligned}
\int_{0}^{1} \frac{b}{2} \ln \left(\frac{a}{b}\right)(t-1)\left(\frac{a}{b}\right)^{t / 2} f\left(a^{1-t}(a b)^{t / 2}\right) f^{\prime}\left(b^{1-t}(a b)^{t / 2}\right) \\
+\frac{a}{2} \ln \left(\frac{b}{a}\right)(t-1)\left(\frac{b}{a}\right)^{t / 2} \\
\quad \times f^{\prime}\left(a^{1-t}(a b)^{t / 2}\right) f\left(b^{1-t}(a b)^{t / 2}\right) d t \\
=\int_{0}^{1}(t-1)\left[f\left(a^{1-t}(a b)^{t / 2}\right) f\left(b^{1-t}(a b)^{t / 2}\right)\right]^{\prime} d t \\
=\left.(t-1) f\left(a^{1-t}(a b)^{t / 2}\right) f\left(b^{1-t}(a b)^{t / 2}\right)\right|_{0} ^{1} \\
\quad-\int_{0}^{1} f\left(a^{1-t}(a b)^{t / 2}\right) f\left(b^{1-t}(a b)^{t / 2}\right) d t \\
=f(a) f(b)-\frac{2}{\ln b-\ln a} \int_{a}^{\sqrt{a b}} \frac{f(x)}{x} f\left(\frac{a b}{x}\right) d x .
\end{aligned}
$$

By the following equality, we obtain inequality (8):

$$
\begin{gathered}
\int_{a}^{\sqrt{a b}} \frac{f(x)}{x} f\left(\frac{a b}{x}\right) d x=\int_{\sqrt{a b}}^{b} \frac{f(x)}{x} f\left(\frac{a b}{x}\right) d x \\
\int_{0}^{1} \frac{b}{2} \ln \left(\frac{a}{b}\right) t\left(\frac{a}{b}\right)^{t / 2} f\left(a^{1-t}(a b)^{t / 2}\right) f^{\prime}\left(b^{1-t}(a b)^{t / 2}\right) \\
\quad+\frac{a}{2} \ln \left(\frac{b}{a}\right) t\left(\frac{b}{a}\right)^{t / 2} \\
\quad \times f^{\prime}\left(a^{1-t}(a b)^{t / 2}\right) f\left(b^{1-t}(a b)^{t / 2}\right) d t \\
=\int_{0}^{1} t\left[f\left(a^{1-t}(a b)^{t / 2}\right) f\left(b^{1-t}(a b)^{t / 2}\right)\right]^{\prime} d t \\
=\left.\quad t f\left(a^{1-t}(a b)^{t / 2}\right) f\left(b^{1-t}(a b)^{t / 2}\right)\right|_{0} ^{1} \\
\quad-\int_{0}^{1} f\left(a^{1-t}(a b)^{t / 2}\right) f\left(b^{1-t}(a b)^{t / 2}\right) d t \\
=f^{2}(\sqrt{a b})-\frac{1}{\ln b-\ln a} \int_{a}^{b} \frac{f(x)}{x} f\left(\frac{a b}{x}\right) d x \\
f^{\sqrt{a b}}\left(\frac{1}{\ln b-\ln a} \int_{a} \frac{2}{x} f\left(\frac{a b}{x}\right) d x\right.
\end{gathered}
$$

This completes the proof of Lemma 7. 
Theorem 8. Let $f: I \subseteq \mathbb{R}_{+} \rightarrow \mathbb{R}_{+}$be differentiable on $I^{\circ}$ and $a, b \in I^{\circ}$ with $a<b$ and $f^{\prime} \in L[a, b]$. If $\left|f^{\prime}\right|^{q}$ is s-geometrically convex on $[a, b]$, for $q \geq 1$ and $s \in(0,1]$, then

$$
\begin{aligned}
& \left|f(a) f(b)-\frac{1}{\ln b-\ln a} \int_{a}^{b} \frac{f(x)}{x} f\left(\frac{a b}{x}\right) d x\right| \\
& \quad \leq \ln \left(\frac{b}{a}\right)\left(\frac{1}{2}\right)^{2-(1 / q)} H_{1}\left(s, q ; h_{1}(\theta), h_{1}(\vartheta)\right), \\
& \left|f^{2}(\sqrt{a b})-\frac{1}{\ln b-\ln a} \int_{a}^{b} \frac{f(x)}{x} f\left(\frac{a b}{x}\right) d x\right| \\
& \quad \leq \ln \left(\frac{b}{a}\right)\left(\frac{1}{2}\right)^{2-(1 / q)} H_{2}\left(s, q ; h_{2}(\theta), h_{2}(\vartheta)\right),
\end{aligned}
$$

where $M_{1}=\max |f(x)|_{x \in[a, \sqrt{a b}]}, M_{2}=\max |f(x)|_{x \in[\sqrt{a b}, b]}$,

$$
\begin{gathered}
h_{1}(u)= \begin{cases}\frac{1}{2}, & u=1, \\
\frac{u-\ln u-1}{(\ln u)^{2}}, & u \neq 1,\end{cases} \\
h_{2}(u)= \begin{cases}\frac{1}{2}, & u=1, \\
\frac{u \ln u-u+1}{(\ln u)^{2}}, & u \neq 1,\end{cases} \\
\theta=\left(\frac{a\left|f^{\prime}(a)\right|^{s}}{b\left|f^{\prime}(b)\right|^{s}}\right)^{q / 2}, \quad \vartheta=\left(\frac{b\left|f^{\prime}(b)\right|^{s}}{a\left|f^{\prime}(a)\right|^{s}}\right)^{q / 2},
\end{gathered}
$$

$$
H_{i}\left(s, q ; h_{i}(\theta), h_{i}(\vartheta)\right)
$$

$$
=\left\{\begin{array}{l}
b\left|f^{\prime}(b)\right|^{s} M_{1} h_{i}^{1 / q}(\theta)+a\left|f^{\prime}(a)\right|^{s} M_{2} h_{i}^{1 / q}(\vartheta), \\
\left|f^{\prime}(a)\right|,\left|f^{\prime}(b)\right| \leq 1 \\
b\left|f^{\prime}(b)\right|\left|f^{\prime}(a)\right|^{1-s} M_{1} h_{i}^{1 / q}(\theta) \\
\quad+a\left|f^{\prime}(a)\right|\left|f^{\prime}(b)\right|^{1-s} M_{2} h_{i}^{1 / q}(\vartheta) \\
\left|f^{\prime}(a)\right|,\left|f^{\prime}(b)\right| \geq 1 \\
b\left|f^{\prime}(b)\right| M_{1} h_{i}^{1 / q}(\theta) \\
\quad+\left.a\left|f^{\prime}(a)\right|\right|^{s}\left|f^{\prime}(b)\right|^{1-s} M_{2} h_{i}^{1 / q}(\vartheta) \\
\left|f^{\prime}(a)\right| \leq 1 \leq\left|f^{\prime}(b)\right| \\
b\left|f^{\prime}(b)\right|^{s}\left|f^{\prime}(a)\right|^{1-s} M_{1} h_{i}^{1 / q}(\theta) \\
\quad+a\left|f^{\prime}(a)\right| M_{2} h_{i}^{1 / q}(\vartheta) \\
\left|f^{\prime}(b)\right| \leq 1 \leq\left|f^{\prime}(a)\right|
\end{array}\right.
$$$$
i=1,2 \text {. }
$$

Proof. (1) Let $M_{1}=\max |f(x)|_{x \in[a, \sqrt{a b}]}, M_{2}=$ $\max |f(x)|_{x \in[\sqrt{a b}, b]}$. Since $\left|f^{\prime}\right|^{q}$ is $s$-geometrically convex on $[a, b]$, from Lemma 7 and power mean inequality, we have

$$
\begin{aligned}
\mid f(a) f(b) & -\frac{1}{\ln b-\ln a} \int_{a}^{b} \frac{f(x)}{x} f\left(\frac{a b}{x}\right) d x \mid \\
\leq \int_{0}^{1} & \frac{b}{2}\left|\ln \left(\frac{a}{b}\right)\right||t-1|\left(\frac{a}{b}\right)^{t / 2} \\
\times & \left|f\left(a^{1-t}(a b)^{t / 2}\right)\right|\left|f^{\prime}\left(b^{1-t}(a b)^{t / 2}\right)\right| \\
+ & \frac{a}{2} \ln \left(\frac{b}{a}\right)|t-1|\left(\frac{b}{a}\right)^{t / 2} \\
& \times\left|f^{\prime}\left(a^{1-t}(a b)^{t / 2}\right)\right|\left|f\left(b^{1-t}(a b)^{t / 2}\right)\right| d t
\end{aligned}
$$$$
\leq \frac{b}{2}\left|\ln \left(\frac{a}{b}\right)\right| M_{1}
$$$$
\times \int_{0}^{1}(1-t)\left(\frac{a}{b}\right)^{t / 2}\left|f^{\prime}\left(b^{1-t}(a b)^{t / 2}\right)\right| d t
$$$$
+\frac{a}{2} \ln \left(\frac{b}{a}\right) M_{2}
$$$$
\times \int_{0}^{1}(1-t)\left(\frac{b}{a}\right)^{t / 2}\left|f^{\prime}\left(a^{1-t}(a b)^{t / 2}\right)\right| d t
$$$$
\leq \frac{b}{2}\left|\ln \left(\frac{a}{b}\right)\right| M_{1}\left(\int_{0}^{1}(1-t) d t\right)^{1-(1 / q)}
$$$$
\times\left(\int_{0}^{1}(1-t)\left(\frac{a}{b}\right)^{q t / 2}\left|f^{\prime}\left(b^{1-t}(a b)^{t / 2}\right)\right|^{q} d t\right)^{1 / q}
$$$$
+\frac{a}{2} \ln \left(\frac{b}{a}\right) M_{2}\left(\int_{0}^{1}(1-t) d t\right)^{1-(1 / q)}
$$$$
\times\left(\int_{0}^{1}(1-t)\left(\frac{b}{a}\right)^{q t / 2}\left|f^{\prime}\left(a^{1-t}(a b)^{t / 2}\right)\right|^{q} d t\right)^{1 / q}
$$$$
\leq \frac{b \ln (b / a) M_{1}}{2}\left(\frac{1}{2}\right)^{1-(1 / q)}
$$$$
\times\left(\int_{0}^{1}(1-t)\left(\frac{a}{b}\right)^{q t / 2}\right.
$$

$$
\left.\times\left|f^{\prime}(a)\right|^{q(t / 2)^{s}}\left|f^{\prime}(b)\right|^{q((2-t) / 2)^{s}} d t\right)^{1 / q}
$$

$$
\begin{aligned}
& +\frac{a \ln (b / a) M_{2}}{2}\left(\frac{1}{2}\right)^{1-(1 / q)} \\
& \times\left(\int_{0}^{1}(1-t)\left(\frac{b}{a}\right)^{q t / 2}\right. \\
& \left.\quad \times\left|f^{\prime}(b)\right|^{q(t / 2)^{s}}\left|f^{\prime}(a)\right|^{q((2-t) / 2)^{s}} d t\right)^{1 / q} .
\end{aligned}
$$


If $0<\mu \leq 1 \leq \eta, 0<t, s \leq 1$, then

$$
\mu^{t^{s}} \leq \mu^{t s}, \quad \eta^{t^{s}} \leq \eta^{t s+1-s}
$$

(i) If $1 \geq\left|f^{\prime}(a)\right|,\left|f^{\prime}(b)\right|$, by (18), we obtain that

$$
\begin{aligned}
\int_{0}^{1}(1 & -t)\left(\frac{a}{b}\right)^{q t / 2}\left|f^{\prime}(a)\right|^{q(t / 2)^{s}}\left|f^{\prime}(b)\right|^{q((2-t) / 2)^{s}} d t \\
& \leq \int_{0}^{1}(1-t)\left(\frac{a}{b}\right)^{q t / 2}\left|f^{\prime}(a)\right|^{q s t / 2}\left|f^{\prime}(b)\right|^{q(q s(2-t) / 2)} d t \\
& =\left|f^{\prime}(b)\right|^{q s} h_{1}(\theta), \\
\int_{0}^{1}(1 & -t)\left(\frac{b}{a}\right)^{q t / 2}\left|f^{\prime}(b)\right|^{q(t / 2)^{s}}\left|f^{\prime}(a)\right|^{q((2-t) / 2)^{s}} d t \\
& \leq \int_{0}^{1}(1-t)\left(\frac{b}{a}\right)^{q t / 2}\left|f^{\prime}(b)\right|^{q s t / 2}\left|f^{\prime}(a)\right|^{q s(2-t) / 2} d t \\
& =\left|f^{\prime}(a)\right|^{q s} h_{1}(\vartheta) .
\end{aligned}
$$

(ii) If $1 \leq\left|f^{\prime}(a)\right|,\left|f^{\prime}(b)\right|$, by (18), we obtain that

$$
\begin{aligned}
\int_{0}^{1}(1 & -t)\left(\frac{a}{b}\right)^{q t / 2}\left|f^{\prime}(a)\right|^{q(t / 2)^{s}}\left|f^{\prime}(b)\right|^{q((2-t) / 2)^{s}} d t \\
\leq & \int_{0}^{1}(1-t)\left(\frac{a}{b}\right)^{q t / 2} \\
& \times\left|f^{\prime}(a)\right|^{q((s t / 2)+1-s)}\left|f^{\prime}(b)\right|^{q(1-(s t / 2))} d t \\
= & \left(\left|f^{\prime}(a)\right|^{1-s}\left|f^{\prime}(b)\right|\right)^{q} h_{1}(\theta), \\
\int_{0}^{1}(1- & t)\left(\frac{b}{a}\right)^{q t / 2}\left|f^{\prime}(b)\right|^{q(t / 2)^{s}}\left|f^{\prime}(a)\right|^{q((2-t) / 2)^{s}} d t \\
\leq & \int_{0}^{1}(1-t)\left(\frac{b}{a}\right)^{q t / 2} \\
& \times\left|f^{\prime}(b)\right|^{q((s t / 2)+1-s)}\left|f^{\prime}(a)\right|^{q(1-(s t / 2))} d t \\
= & \left(\left|f^{\prime}(b)\right|^{1-s}\left|f^{\prime}(a)\right|\right)^{q} h_{1}(\vartheta) .
\end{aligned}
$$

(iii) If $\left|f^{\prime}(a)\right| \leq 1 \leq\left|f^{\prime}(b)\right|$, by (18), we obtain that

$$
\begin{aligned}
\int_{0}^{1}(1 & -t)\left(\frac{a}{b}\right)^{q t / 2}\left|f^{\prime}(a)\right|^{q(t / 2)^{s}}\left|f^{\prime}(b)\right|^{q((2-t) / 2)^{s}} d t \\
& \leq \int_{0}^{1}(1-t)\left(\frac{a}{b}\right)^{q t / 2}\left|f^{\prime}(a)\right|^{q s t / 2}\left|f^{\prime}(b)\right|^{q(1-(s t / 2))} d t \\
& =\left|f^{\prime}(b)\right|^{q} h_{1}(\theta),
\end{aligned}
$$

$$
\begin{aligned}
\int_{0}^{1}(1 & -t)\left(\frac{b}{a}\right)^{q t / 2}\left|f^{\prime}(b)\right|^{q(t / 2)^{s}}\left|f^{\prime}(a)\right|^{q((2-t) / 2)^{s}} d t \\
& \leq \int_{0}^{1}(1-t)\left(\frac{b}{a}\right)^{q t / 2}\left|f^{\prime}(b)\right|^{q((s t / 2)+1-s)}\left|f^{\prime}(a)\right|^{q s(2-t) / 2} d t \\
& =\left(\left|f^{\prime}(a)\right|^{s}\left|f^{\prime}(b)\right|^{1-s}\right)^{q} h_{1}(\vartheta) .
\end{aligned}
$$

(iv) If $\left|f^{\prime}(b)\right| \leq 1 \leq\left|f^{\prime}(a)\right|$, by (18), we obtain that

$$
\begin{aligned}
\int_{0}^{1}(1 & -t)\left(\frac{a}{b}\right)^{q t / 2}\left|f^{\prime}(a)\right|^{q(t / 2)^{s}}\left|f^{\prime}(b)\right|^{q((2-t) / 2)^{s}} d t \\
& \leq \int_{0}^{1}(1-t)\left(\frac{a}{b}\right)^{q t / 2}\left|f^{\prime}(a)\right|^{q((s t / 2)+1-s)}\left|f^{\prime}(b)\right|^{q s(2-t) / 2} d t \\
& =\left(\left|f^{\prime}(a)\right|^{1-s}\left|f^{\prime}(b)\right|^{s}\right)^{q} h_{1}(\theta), \\
\int_{0}^{1}(1 & -t)\left(\frac{b}{a}\right)^{q t / 2}\left|f^{\prime}(b)\right|^{q(t / 2)^{s}}\left|f^{\prime}(a)\right|^{q((2-t) / 2)^{s}} d t \\
& \leq \int_{0}^{1}(1-t)\left(\frac{b}{a}\right)^{q t / 2}\left|f^{\prime}(b)\right|^{q s t / 2}\left|f^{\prime}(a)\right|^{q(1-(s t / 2))} d t \\
& =\left|f^{\prime}(a)\right|^{q} h_{1}(\vartheta) .
\end{aligned}
$$

From (17) to (22), (12) holds.

(2) Let $M_{1}=\max |f(x)|_{x \in[a, \sqrt{a b}]}, M_{2}=$ $\max |f(x)|_{x \in[\sqrt{a b}, b]}$. Since $\left|f^{\prime}\right|^{q}$ is $s$-geometrically convex on $[a, b]$, from Lemma 7 and Hölder inequality, we have

$$
\begin{aligned}
\mid f^{2}(\sqrt{a b}) & -\frac{1}{\ln b-\ln a} \int_{a}^{b} \frac{f(x)}{x} f\left(\frac{a b}{x}\right) d x \mid \\
\leq & \int_{0}^{1} \frac{b}{2}\left|\ln \left(\frac{a}{b}\right)\right| t\left(\frac{a}{b}\right)^{t / 2} \\
& \times\left|f\left(a^{1-t}(a b)^{t / 2}\right)\right|\left|f^{\prime}\left(b^{1-t}(a b)^{t / 2}\right)\right| \\
& +\frac{a}{2} \ln \left(\frac{b}{a}\right) t\left(\frac{b}{a}\right)^{t / 2} \\
& \times\left|f^{\prime}\left(a^{1-t}(a b)^{t / 2}\right)\right|\left|f\left(b^{1-t}(a b)^{t / 2}\right)\right| d t \\
\leq & \frac{b}{2} \ln \left(\frac{b}{a}\right) M_{1} \int_{0}^{1} t\left(\frac{a}{b}\right)^{t / 2}\left|f^{\prime}\left(b^{1-t}(a b)^{t / 2}\right)\right| d t \\
& +\frac{a}{2} \ln \left(\frac{b}{a}\right) M_{2} \int_{0}^{1} t\left(\frac{b}{a}\right)^{t / 2}\left|f^{\prime}\left(a^{1-t}(a b)^{t / 2}\right)\right| d t \\
\leq & \frac{b}{2} \ln \left(\frac{b}{a}\right) M_{1}\left(\int_{0}^{1} t d t\right)^{1-(1 / q)}
\end{aligned}
$$




$$
\begin{aligned}
& \times\left(\int_{0}^{1} t\left(\frac{a}{b}\right)^{q t / 2}\left|f^{\prime}\left(b^{1-t}(a b)^{t / 2}\right)\right|^{q} d t\right)^{1 / q} \\
& +\frac{a}{2} \ln \left(\frac{b}{a}\right) M_{2}\left(\int_{0}^{1} t d t\right)^{1-(1 / q)} \\
& \times\left(\int_{0}^{1} t\left(\frac{b}{a}\right)^{q t / 2}\left|f^{\prime}\left(a^{1-t}(a b)^{t / 2}\right)\right|^{q} d t\right)^{1 / q} \\
& \left.\left.\left.\leq \frac{b \ln (b / a) M_{1}}{2}\left(\frac{1}{2}\right)^{1-(1 / q)}\right)^{q}\right)^{q t / 2}\left|f^{\prime}(a)\right|^{q(t / 2)^{s}}\left|f^{\prime}(b)\right|^{q((2-t) / 2)^{s}} d t\right)^{1 / q} \\
& \times\left(\int_{0}^{1} t\left(\frac{a}{b}\right)^{1-(1 / q)}\right. \\
& +\frac{a \ln (b / a) M_{2}}{2}\left(\frac{1}{2}\right)^{1 / q} \\
& \times\left(\int_{0}^{1} t\left(\frac{b}{a}\right)^{q t / 2}\left|f^{\prime}(b)\right|^{q(t / 2)^{s}}\left|f^{\prime}(a)\right|^{q((2-t) / 2)^{s}} d t\right)^{1 / q} .
\end{aligned}
$$

(i) If $1 \geq\left|f^{\prime}(a)\right|,\left|f^{\prime}(b)\right|$, by (18), we obtain that

$$
\begin{aligned}
& \int_{0}^{1} t\left(\frac{a}{b}\right)^{q t / 2}\left|f^{\prime}(a)\right|^{q(t / 2)^{s}}\left|f^{\prime}(b)\right|^{q((2-t) / 2)^{s}} d t \\
& \leq\left|f^{\prime}(b)\right|^{q s} h_{2}(\theta), \\
& \int_{0}^{1} t\left(\frac{b}{a}\right)^{q t / 2}\left|f^{\prime}(b)\right|^{q(t / 2)^{s}}\left|f^{\prime}(a)\right|^{q((2-t) / 2)^{s}} d t \\
& \leq\left|f^{\prime}(a)\right|^{q s} h_{2}(\vartheta) .
\end{aligned}
$$

(ii) If $1 \leq\left|f^{\prime}(a)\right|,\left|f^{\prime}(b)\right|$, by (18), we obtain that

$$
\begin{gathered}
\int_{0}^{1} t\left(\frac{a}{b}\right)^{q t / 2}\left|f^{\prime}(a)\right|^{q(t / 2)^{s}}\left|f^{\prime}(b)\right|^{q((2-t) / 2)^{s}} d t \\
\leq\left(\left|f^{\prime}(a)\right|^{1-s}\left|f^{\prime}(b)\right|\right)^{q} h_{2}(\theta), \\
\int_{0}^{1} t\left(\frac{b}{a}\right)^{q t / 2}\left|f^{\prime}(b)\right|^{q(t / 2)^{s}}\left|f^{\prime}(a)\right|^{q((2-t) / 2)^{s}} d t \\
\leq\left(\left|f^{\prime}(b)\right|^{1-s}\left|f^{\prime}(a)\right|\right)^{q} h_{2}(\vartheta) .
\end{gathered}
$$

(iii) If $\left|f^{\prime}(a)\right| \leq 1 \leq\left|f^{\prime}(b)\right|$, by (18), we obtain that

$$
\begin{aligned}
& \int_{0}^{1} t\left(\frac{a}{b}\right)^{q t / 2}\left|f^{\prime}(a)\right|^{q(t / 2)^{s}}\left|f^{\prime}(b)\right|^{q((2-t) / 2)^{s}} d t \\
& \leq\left|f^{\prime}(b)\right|^{q} h_{2}(\theta), \\
& \int_{0}^{1} t\left(\frac{b}{a}\right)^{q t / 2}\left|f^{\prime}(b)\right|^{q(t / 2)^{s}}\left|f^{\prime}(a)\right|^{q((2-t) / 2)^{s}} d t \\
& \leq\left(\left|f^{\prime}(a)\right|^{s}\left|f^{\prime}(b)\right|^{1-s}\right)^{q} h_{2}(\vartheta) .
\end{aligned}
$$

(iv) If $\left|f^{\prime}(b)\right| \leq 1 \leq\left|f^{\prime}(a)\right|$, by (18), we obtain that

$$
\begin{aligned}
& \int_{0}^{1} t\left(\frac{a}{b}\right)^{q t / 2}\left|f^{\prime}(a)\right|^{q(t / 2)^{s}}\left|f^{\prime}(b)\right|^{q((2-t) / 2)^{s}} d t \\
& \leq\left(\left|f^{\prime}(a)\right|^{1-s}\left|f^{\prime}(b)\right|^{s}\right)^{q} h_{2}(\theta), \\
& \int_{0}^{1} t\left(\frac{b}{a}\right)^{q t / 2}\left|f^{\prime}(b)\right|^{q(t / 2)^{s}}\left|f^{\prime}(a)\right|^{q((2-t) / 2)^{s}} d t \\
& \leq\left|f^{\prime}(a)\right|^{q} h_{2}(\vartheta) .
\end{aligned}
$$

From (23) to (27), (13) holds. This completes the required proof.

If we take $s=1$ in Theorem 8 , we can derive the following corollary.

Corollary 9. Let $f: I \subseteq \mathbb{R}_{+} \rightarrow \mathbb{R}_{+}$be differentiable on $I^{\circ}$ and $a, b \in I^{\circ}$ with $a<b$ and $f^{\prime} \in L[a, b]$. If $\left|f^{\prime}\right|^{q}$ is geometrically convex on $[a, b]$, for $q \geq 1$, then

$$
\begin{aligned}
& \left|f(a) f(b)-\frac{1}{\ln b-\ln a} \int_{a}^{b} \frac{f(x)}{x} f\left(\frac{a b}{x}\right) d x\right| \\
& \quad \leq \ln \left(\frac{b}{a}\right)\left(\frac{1}{2}\right)^{2-(1 / q)} H_{1}\left(1, q ; h_{1}(\theta), h_{1}(\vartheta)\right), \\
& \left|f^{2}(\sqrt{a b})-\frac{1}{\ln b-\ln a} \int_{a}^{b} \frac{f(x)}{x} f\left(\frac{a b}{x}\right) d x\right| \\
& \leq \ln \left(\frac{b}{a}\right)\left(\frac{1}{2}\right)^{2-(1 / q)} H_{2}\left(1, q ; h_{2}(\theta), h_{2}(\vartheta)\right),
\end{aligned}
$$

where $\theta, \vartheta, H_{1}, H_{2}, h_{1}$, and $h_{2}$ are the same as in Theorem 8 .

If we take $q=1$ in Theorem 8 , we can derive the following corollary.

Corollary 10. Let $f: I \subseteq \mathbb{R}_{+} \rightarrow \mathbb{R}_{+}$be differentiable on $I^{\circ}$ and $a, b \in I^{\circ}$ with $a<b$ and $f^{\prime} \in L[a, b]$. If $\left|f^{\prime}\right|$ is $s$ geometrically convex on $[a, b]$, for $s \in(0,1]$, then

$$
\begin{gathered}
\left|f(a) f(b)-\frac{1}{\ln b-\ln a} \int_{a}^{b} \frac{f(x)}{x} f\left(\frac{a b}{x}\right) d x\right| \\
\leq \ln \left(\frac{b}{a}\right)\left(\frac{1}{2}\right) H_{1}\left(s, 1 ; h_{1}(\theta), h_{1}(\vartheta)\right), \\
\left|f^{2}(\sqrt{a b})-\frac{1}{\ln b-\ln a} \int_{a}^{b} \frac{f(x)}{x} f\left(\frac{a b}{x}\right) d x\right| \\
\leq \ln \left(\frac{b}{a}\right)\left(\frac{1}{2}\right) H_{2}\left(s, 1 ; h_{2}(\theta), h_{2}(\vartheta)\right),
\end{gathered}
$$

where $\theta, \vartheta, H_{1}, H_{2}, h_{1}$, and $h_{2}$ are the same as in Theorem 8 . 
Theorem 11. Let $f: I \subseteq \mathbb{R}_{+} \rightarrow \mathbb{R}_{+}$be differentiable on $I^{\circ}$ and $a, b \in I^{\circ}$ with $a<b$ and $f^{\prime} \in L[a, b]$. If $\left|f^{\prime}\right|^{q}$ is s-geometrically convex on $[a, b]$, for $q>1$ and $s \in(0,1]$, then

$$
\begin{aligned}
& \left|f(a) f(b)-\frac{1}{\ln b-\ln a} \int_{a}^{b} \frac{f(x)}{x} f\left(\frac{a b}{x}\right) d x\right| \\
& \quad \leq \frac{\ln (b / a)}{2}\left(\frac{q-1}{2 q-1}\right)^{1-(1 / q)} H_{3}\left(s, q ; h_{3}(\theta), h_{3}(\vartheta)\right), \\
& \left|f^{2}(\sqrt{a b})-\frac{1}{\ln b-\ln a} \int_{a}^{b} \frac{f(x)}{x} f\left(\frac{a b}{x}\right) d x\right| \\
& \quad \leq \frac{\ln (b / a)}{2}\left(\frac{q-1}{2 q-1}\right)^{1-(1 / q)} H_{3}\left(s, q ; h_{3}(\theta), h_{3}(\vartheta)\right),
\end{aligned}
$$

where $M_{1}=\max |f(x)|_{x \in[a, \sqrt{a b}]}, M_{2}=\max |f(x)|_{x \in[\sqrt{a b}, b]}$,

$$
h_{3}(u)=\left\{\begin{array}{ll}
1, & u=1, \\
\frac{u-1}{\ln u}, & u \neq 1,
\end{array} \quad u>0,\right.
$$

$H_{3}\left(s, q ; h_{3}(\theta), h_{3}(\vartheta)\right)$

$$
=\left\{\begin{array}{l}
b\left|f^{\prime}(b)\right|^{s} M_{1} h_{3}^{1 / q}(\theta)+a\left|f^{\prime}(a)\right|^{s} M_{2} h_{3}^{1 / q}(\vartheta), \\
\left|f^{\prime}(a)\right|,\left|f^{\prime}(b)\right| \leq 1 \\
b\left|f^{\prime}(b)\right|\left|f^{\prime}(a)\right|^{1-s} M_{1} h_{3}^{1 / q}(\theta) \\
\quad+a\left|f^{\prime}(a)\right|\left|f^{\prime}(b)\right|^{1-s} M_{2} h_{3}^{1 / q}(\vartheta) \\
\left|f^{\prime}(a)\right|,\left|f^{\prime}(b)\right| \geq 1 \\
b\left|f^{\prime}(b)\right| M_{1} h_{3}^{1 / q}(\theta) \\
\quad+a\left|f^{\prime}(a)\right|^{s}\left|f^{\prime}(b)\right|^{1-s} M_{2} h_{3}^{1 / q}(\vartheta) \\
\left|f^{\prime}(a)\right| \leq 1 \leq\left|f^{\prime}(b)\right| \\
b\left|f^{\prime}(b)\right|^{s} M_{1} h_{3}^{1 / q}(\theta)+a\left|f^{\prime}(a)\right| M_{2} h_{3}^{1 / q}(\vartheta), \\
\left|f^{\prime}(b)\right| \leq 1 \leq\left|f^{\prime}(a)\right|,
\end{array}\right.
$$

and $\theta, 9$ are the same as in (15).

Proof. (1) Let $M_{1}=\max |f(x)|_{x \in[a, \sqrt{a b}]}, M_{2}=$ $\max |f(x)|_{x \in[\sqrt{a b}, b]}$. Since $\left|f^{\prime}\right|^{q}$ is $s$-geometrically convex on $[a, b]$, from Lemma 7 and Hölder inequality, we have

$$
\begin{aligned}
& \mid f(a) f(b)-\frac{1}{\ln b-\ln a} \int_{a}^{b} \frac{f(x)}{x} f\left(\frac{a b}{x}\right) d x \mid \\
& \leq \int_{0}^{1} \frac{b}{2}\left|\ln \left(\frac{a}{b}\right)\right||t-1|\left(\frac{a}{b}\right)^{t / 2} \\
& \times\left|f\left(a^{1-t}(a b)^{t / 2}\right)\right|\left|f^{\prime}\left(b^{1-t}(a b)^{t / 2}\right)\right| \\
&+\frac{a}{2} \ln \left(\frac{b}{a}\right)|t-1|\left(\frac{b}{a}\right)^{t / 2} \\
& \times\left|f^{\prime}\left(a^{1-t}(a b)^{t / 2}\right)\right|\left|f\left(b^{1-t}(a b)^{t / 2}\right)\right| d t
\end{aligned}
$$

$$
\begin{aligned}
\leq & \frac{b}{2}\left|\ln \left(\frac{a}{b}\right)\right| M_{1} \\
& \times \int_{0}^{1}(1-t)\left(\frac{a}{b}\right)^{t / 2}\left|f^{\prime}\left(b^{1-t}(a b)^{t / 2}\right)\right| d t \\
& +\frac{a}{2} \ln \left(\frac{b}{a}\right) M_{2} \\
& \times \int_{0}^{1}(1-t)\left(\frac{b}{a}\right)^{t / 2}\left|f^{\prime}\left(a^{1-t}(a b)^{t / 2}\right)\right| d t \\
\leq & \frac{b}{2}\left|\ln \left(\frac{a}{b}\right)\right| M_{1}\left(\int_{0}^{1}(1-t)^{q /(q-1)} d t\right)^{1-(1 / q)} \\
& \times\left(\int_{0}^{1}\left(\frac{a}{b}\right)^{q t / 2}\left|f^{\prime}\left(b^{1-t}(a b)^{t / 2}\right)\right|^{q} d t\right)^{1 / q} \\
& +\frac{a}{2} \ln \left(\frac{b}{a}\right) M_{2}\left(\int_{0}^{1}(1-t)^{q /(q-1)} d t\right)^{1-(1 / q)} \\
& \times\left(\int_{0}^{1}\left(\frac{b}{a}\right)^{q t / 2}\left|f^{\prime}\left(a^{1-t}(a b)^{t / 2}\right)\right|^{q} d t\right)^{1 / q} \\
\leq & \frac{b \ln (b / a) M_{1}}{2}\left(\frac{q-1}{2 q-1}\right)^{1-(1 / q)} \\
\times & \left.\times\left(\int_{0}^{1}\left(\frac{b}{a}\right)^{q t / 2}\left|f^{\prime}(b)\right|^{q(t / 2)^{s}}\left|f^{\prime}(a)\right|^{q((2-t) / 2)^{s}} d t\right)^{q t / 2}\left|f^{\prime}(a)\right|^{q(t / 2)^{s}}\left|f^{\prime}(b)\right|^{q((2-t) / 2)^{s}} d t\right)^{1 / q} \\
& +\frac{a \ln (b / a) M_{2}}{2}\left(\frac{q-1}{2 q-1}\right)^{1-(1 / q)}
\end{aligned}
$$

(i) If $1 \geq\left|f^{\prime}(a)\right|,\left|f^{\prime}(b)\right|$, by (18), we have

$$
\begin{aligned}
& \int_{0}^{1}\left(\frac{a}{b}\right)^{q t / 2}\left|f^{\prime}(a)\right|^{q(t / 2)^{s}}\left|f^{\prime}(b)\right|^{q((2-t) / 2)^{s}} d t \\
& =\left|f^{\prime}(b)\right|^{q s} h_{3}(\theta), \\
& \int_{0}^{1}\left(\frac{b}{a}\right)^{q t / 2}\left|f^{\prime}(b)\right|^{q(t / 2)^{s}}\left|f^{\prime}(a)\right|^{q((2-t) / 2)^{s}} d t \\
& =\left|f^{\prime}(a)\right|^{q s} h_{3}(9) .
\end{aligned}
$$

(ii) If $1 \leq\left|f^{\prime}(a)\right|,\left|f^{\prime}(b)\right|$, by (18), we have

$$
\begin{gathered}
\int_{0}^{1}\left(\frac{a}{b}\right)^{q t / 2}\left|f^{\prime}(a)\right|^{q(t / 2)^{s}}\left|f^{\prime}(b)\right|^{q((2-t) / 2)^{s}} d t \\
\leq\left(\left|f^{\prime}(b)\right|\left|f^{\prime}(a)\right|^{1-s}\right)^{q} h_{3}(\theta),
\end{gathered}
$$




$$
\begin{gathered}
\int_{0}^{1}\left(\frac{b}{a}\right)^{q t / 2}\left|f^{\prime}(b)\right|^{q(t / 2)^{s}}\left|f^{\prime}(a)\right|^{q((2-t) / 2)^{s}} d t \\
\leq\left(\left|f^{\prime}(a)\right|\left|f^{\prime}(b)\right|^{1-s}\right)^{q} h_{3}(\vartheta) .
\end{gathered}
$$

(iii) If $\left|f^{\prime}(a)\right| \leq 1 \leq\left|f^{\prime}(b)\right|$, by (18), we obtain that

$$
\begin{aligned}
& \int_{0}^{1}\left(\frac{a}{b}\right)^{q t / 2}\left|f^{\prime}(a)\right|^{q(t / 2)^{s}}\left|f^{\prime}(b)\right|^{q((2-t) / 2)^{s}} d t \\
& \leq\left|f^{\prime}(b)\right|^{q} h_{3}(\theta), \\
& \int_{0}^{1}\left(\frac{b}{a}\right)^{q t / 2}\left|f^{\prime}(b)\right|^{q(t / 2)^{s}}\left|f^{\prime}(a)\right|^{q((2-t) / 2)^{s}} d t \\
& \leq\left(\left|f^{\prime}(a)\right| f^{s}\left|f^{\prime}(b)\right|^{1-s}\right)^{q} h_{3}(9) .
\end{aligned}
$$

(iv) If $\left|f^{\prime}(b)\right| \leq 1 \leq\left|f^{\prime}(a)\right|$, by (18), we obtain that

$$
\begin{aligned}
& \int_{0}^{1}\left(\frac{a}{b}\right)^{q t / 2}\left|f^{\prime}(a)\right|^{q(t / 2)^{s}}\left|f^{\prime}(b)\right|^{q((2-t) / 2)^{s}} d t \\
& \leq\left(\left|f^{\prime}(a)\right|^{1-s}\left|f^{\prime}(b)\right|^{s}\right)^{q} h_{3}(\theta), \\
& \int_{0}^{1}\left(\frac{b}{a}\right)^{q t / 2}\left|f^{\prime}(b)\right|^{q(t / 2)^{s}}\left|f^{\prime}(a)\right|^{q((2-t) / 2)^{s}} d t \\
& \leq\left|f^{\prime}(a)\right|^{q} h_{3}(9) .
\end{aligned}
$$

From (33) to (37), (30) holds.

(2) Let $M_{1}=\max |f(x)|_{x \in[a, \sqrt{a b}]}, \quad M_{2}=$ $\max |f(x)|_{x \in[\sqrt{a b}, b]}$ Since $\left|f^{\prime}\right|^{q}$ is s-geometrically convex on $[a, b]$, from Lemma 7 and Hölder inequality, we have

$$
\begin{aligned}
\mid f^{2}( & \sqrt{a b})-\frac{1}{\ln b-\ln a} \int_{a}^{b} \frac{f(x)}{x} f\left(\frac{a b}{x}\right) d x \mid \\
\leq & \frac{b}{2} \ln \left(\frac{b}{a}\right) M_{1}\left(\frac{q-1}{2 q-1}\right)^{1-(1 / q)} \\
& \times\left(\int_{0}^{1}\left(\frac{a}{b}\right)^{q t / 2}\left|f^{\prime}(a)\right|^{q(t / 2)^{s}}\left|f^{\prime}(b)\right|^{q((2-t) / 2)^{s}} d t\right)^{1 / q} \\
& +\frac{a}{2} \ln \left(\frac{b}{a}\right) M_{2}\left(\frac{q-1}{2 q-1}\right)^{1-(1 / q)} \\
& \times\left(\int_{0}^{1}\left(\frac{b}{a}\right)^{q t / 2}\left|f^{\prime}(b)\right|^{q(t / 2)^{s}}\left|f^{\prime}(a)\right|^{q((2-t) / 2)^{s}} d t\right)^{1 / q} .
\end{aligned}
$$

From (38) and (34) to (37), (31) holds.

If taking $s=1$ in Theorem 11, we can derive the following corollary.
Corollary 12. Let $f: I \subseteq \mathbb{R}_{+} \rightarrow \mathbb{R}_{+}$be differentiable on $I^{\circ}$ and $a, b \in I^{\circ}$ with $a<b$ and $f^{\prime} \in L[a, b]$. If $\left|f^{\prime}\right|^{q}$ is geometrically convex on $[a, b]$, for $q>1$, then

$$
\begin{aligned}
& \left|f(a) f(b)-\frac{1}{\ln b-\ln a} \int_{a}^{b} \frac{f(x)}{x} f\left(\frac{a b}{x}\right) d x\right| \\
& \quad \leq \frac{1}{2} \ln \left(\frac{b}{a}\right)\left(\frac{q-1}{2 q-1}\right)^{1-(1 / q)} H_{3}\left(1, q ; h_{3}(\theta), h_{3}(9)\right), \\
& \left|f^{2}(\sqrt{a b})-\frac{1}{\ln b-\ln a} \int_{a}^{b} \frac{f(x)}{x} f\left(\frac{a b}{x}\right) d x\right| \\
& \quad \leq \frac{1}{2} \ln \left(\frac{b}{a}\right)\left(\frac{q-1}{2 q-1}\right)^{1-(1 / q)} H_{3}\left(1, q ; h_{3}(\theta), h_{3}(9)\right),
\end{aligned}
$$

where $\theta, \vartheta, H_{3}$, and $h_{3}$ are the same as in Theorem 11.

\section{Application to Special Means}

Let us recall the following special means of two nonnegative numbers $a, b$ with $>a$.

(1) The arithmetic mean

$$
A=A(a, b):=\frac{a+b}{2} .
$$

(2) The geometric mean

$$
G=G(a, b):=\sqrt{a b} .
$$

(3) The logarithmic mean

$$
L=L(a, b):=\frac{b-a}{\ln b-\ln a} .
$$

(4) The $p$-logarithmic mean

$$
L_{p}=L_{p}(a, b):=\left(\frac{b^{p+1}-a^{p+1}}{(p+1)(b-a)}\right)^{1 / p}, \quad p \in \mathbb{R} \backslash\{-1,0\} .
$$

Let $f(x)=\left(x^{s} / s\right)+1, x \in(0,1], 0<s<1, q \geq 1$, and then the function $\left|f^{\prime}(x)\right|^{q}=x^{(s-1) q}$ is $s$-geometrically convex on $(0,1]$ for $0<s<1$ (see [2]).

Proposition 13. Let $0<a<b \leq 1,0<s<1$, and $q \geq 1$. Then

$$
\begin{aligned}
& \mid G^{2}\left(\frac{a^{s}}{s}+1, \frac{b^{s}}{s}+1\right)-\frac{2}{s^{2}} A\left(G^{2}\left(a^{s}, b^{s}\right), s^{2}\right) \\
& -\frac{2}{s} L_{s-1}^{s-1}(a, b) L(a, b) \mid \\
& \leq \frac{1}{G^{(s-1)^{2}}(a, b)}\left(\frac{1}{\left(s^{2}-s+1\right) q}\right)^{1 / q}\left(\frac{b-a}{4 L(a, b)}\right)^{1-(1 / q)} \\
& \quad \times\left[M_{1} G\left(a^{-(s-1)^{2}}, b^{s}\right)\right.
\end{aligned}
$$




$$
\begin{aligned}
& \times\left\{b^{\left(s^{2}-s+1\right) q / 2}-L\left(a^{\left(s^{2}-s+1\right) q / 2}, b^{\left(s^{2}-s+1\right) q / 2}\right)\right\}^{1 / q} \\
& \times M_{2} G\left(b^{-(s-1)^{2}}, a^{s}\right) \\
& \left.\times\left\{L\left(a^{\left(s^{2}-s+1\right) q / 2}, b^{\left(s^{2}-s+1\right) q / 2}\right)-a^{\left(s^{2}-s+1\right) q / 2}\right\}^{1 / q}\right] \\
& \left.\frac{G^{s}(a, b)}{s}+1\right)^{2}-\frac{2}{s^{s}} A\left(G^{2}\left(a^{s}, b^{s}\right), s^{2}\right) \\
-\frac{2}{s} L_{s-1}^{s-1}(a, b) L(a, b) \mid & \\
\leq & \frac{1}{G^{(s-1)^{2}}(a, b)}\left(\frac{1}{\left(s^{2}-s+1\right) q}\right)^{1 / q}\left(\frac{b-a}{4 L(a, b)}\right)^{1-(1 / q)} \\
& \times\left[M_{1} G\left(a^{-(s-1)^{2}}, b^{s}\right)\right. \\
& \times\left\{L\left(a^{\left(s^{2}-s+1\right) q / 2}-L\left(a^{\left(s^{2}-s+1\right) q / 2}, b^{\left(s^{2}-s+1\right) q / 2}\right)\right\}^{1 / q}\right. \\
& \left.\times M_{2} G\left(b^{-(s-1)^{2}}, a^{s}\right)\right] \\
& \\
& \\
& \\
&
\end{aligned}
$$

where $M_{1}=\left(\sqrt{a b}^{s} / s\right)+1$ and $M_{2}=\left(b^{s} / s\right)+1$.

Proof. Let $f(x)=\left(x^{s} / s\right)+1, x \in(0,1], 0<s<1$. Then $\left|f^{\prime}(a)\right|=a^{s-1}>b^{s-1}=\left|f^{\prime}(b)\right| \geq 1, M_{1}=\max |f(x)|_{x \in[a, \sqrt{a b}]}$ $=\left(\sqrt{a b}^{s} / s\right)+1, M_{2}=\max |f(x)|_{x \in[\sqrt{a b}, b]}=\left(b^{s} / s\right)+1$. Thus, by Theorem 8 , Proposition 13 is proved.

Proposition 14. Let $0<a<b \leq 1,0<s<1$, and $q>1$. Then

$$
\begin{aligned}
& \mid G^{2}\left(\frac{a^{s}}{s}+1, \frac{b^{s}}{s}+1\right)-\frac{2}{s^{2}} A\left(G^{2}\left(a^{s}, b^{s}\right), s^{2}\right) \\
& -\frac{2}{s} L_{s-1}^{s-1}(a, b) L(a, b) \mid \\
& \leq \frac{b-a}{2 L(a, b)}\left(\frac{q-1}{2 q-1}\right)^{1-(1 / q)} \\
& \quad \times \frac{L^{1 / q}\left(a^{\left(s^{2}-s+1\right) q / 2}, b^{\left(s^{2}-s+1\right) q / 2}\right)}{G^{(s-1)^{2}}(a, b)} \\
& \quad \times\left\{M_{1} G\left(a^{-(s-1)^{2}}, b^{s}\right)+M_{2} G\left(b^{-(s-1)^{2}}, a^{s}\right)\right\},
\end{aligned}
$$

$$
\begin{aligned}
& \mid\left(\frac{G^{s}(a, b)}{s}+1\right)^{2}-\frac{2}{s^{s}} A\left(G^{2}\left(a^{s}, b^{s}\right), s^{2}\right) \\
& -\frac{2}{s} L_{s-1}^{s-1}(a, b) L(a, b) \mid \\
& \leq \frac{b-a}{2 L(a, b)}\left(\frac{q-1}{2 q-1}\right)^{1-(1 / q)} \\
& \quad \times \frac{L^{1 / q}\left(a^{\left(s^{2}-s+1\right) q / 2}, b^{\left(s^{2}-s+1\right) q / 2}\right)}{G^{(s-1)^{2}}(a, b)} \\
& \quad \times\left\{M_{1} G\left(a^{-(s-1)^{2}}, b^{s}\right)+M_{2} G\left(b^{-(s-1)^{2}}, a^{s}\right)\right\},
\end{aligned}
$$

where $M_{1}=\left(\sqrt{a b}^{s} / s\right)+1$ and $M_{2}=\left(b^{s} / s\right)+1$.

Proof. Let $f(x)=\left(x^{s} / s\right)+1, x \in(0,1], 0<s<1$. Then $\left|f^{\prime}(a)\right|=a^{s-1}>b^{s-1}=\left|f^{\prime}(b)\right| \geq 1, M_{1}=\max |f(x)|_{x \in[a, \sqrt{a b}]}$ $=\left(\sqrt{a b}^{s} / s\right)+1, M_{2}=\max |f(x)|_{x \in[\sqrt{a b}, b]}=\left(b^{s} / s\right)+1$. Thus, by Theorem 11, Proposition 14 is proved.

\section{Conflict of Interests}

The author declares that there is no conflict of interests regarding the publication of this paper.

\section{References}

[1] H. Hudzik and L. Maligranda, "Some remarks on s-convex functions," Aequationes Mathematicae, vol. 48, no. 1, pp. 100111, 1994.

[2] T. Y. Zhang, A. P. Ji, and F. Qi, "On integral inequalities of Hermite-Hadamard type for $s$-geometrically convex functions," Abstract and Applied Analysis, vol. 2012, Article ID 560586, 14 pages, 2012.

[3] M. W. Alomari, M. Darus, and U. S. Kirmaci, "Some inequalities of Hermite-Hadamard type for $s$-convex functions," Acta Mathematica Scientia, vol. 31, no. 4, pp. 1643-1652, 2011.

[4] S. Hussain, M. I. Bhatti, and M. Iqbal, "Hadamard-type inequalities for s-convex functions I," Punjab University Journal of Mathematics, vol. 41, p. 51-60, 2009.

[5] I. Işcan, "New estimates on generalization of some integral inequalities for $s$-convex functions and their applications," International Journal of Pure and Applied Mathematics, vol. 86, no. 4, pp. 727-746, 2013.

[6] U. S. Kirmaci, M. Klari ucic Bakula, M. E. Özdemir, and J. Pe $\mu$ caric, "Hadamard-type inequalities for $s$-convex functions," Applied Mathematics and Computation, vol. 193, no. 1, p. 26-35, 2007.

[7] M. Tunç, "On Hadamard type inequalities for s-geometrically convex functions," RGMIA Research Report Collection, vol. 15, article 70, 6 pages, 2012. 


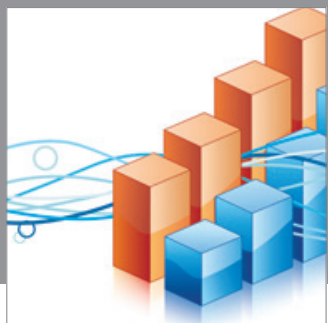

Advances in

Operations Research

mansans

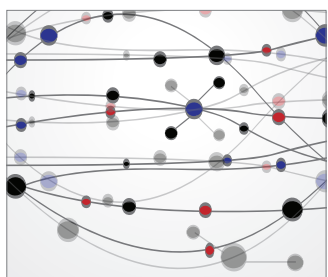

The Scientific World Journal
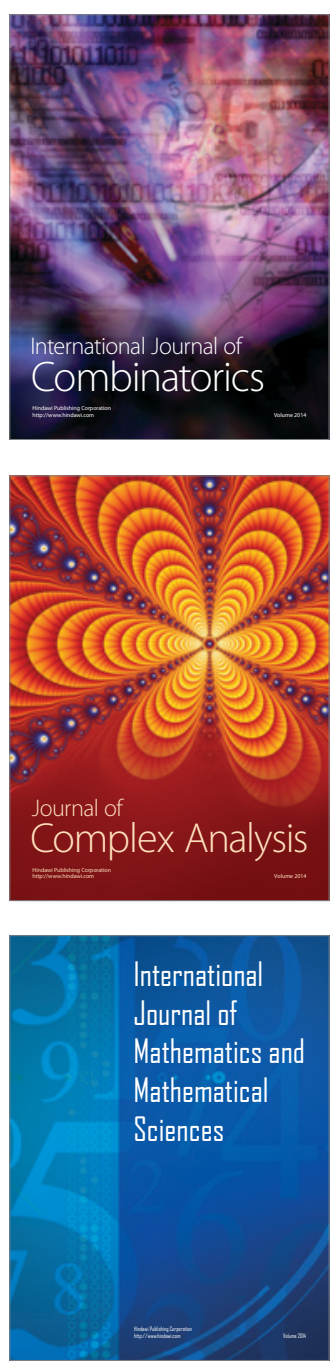
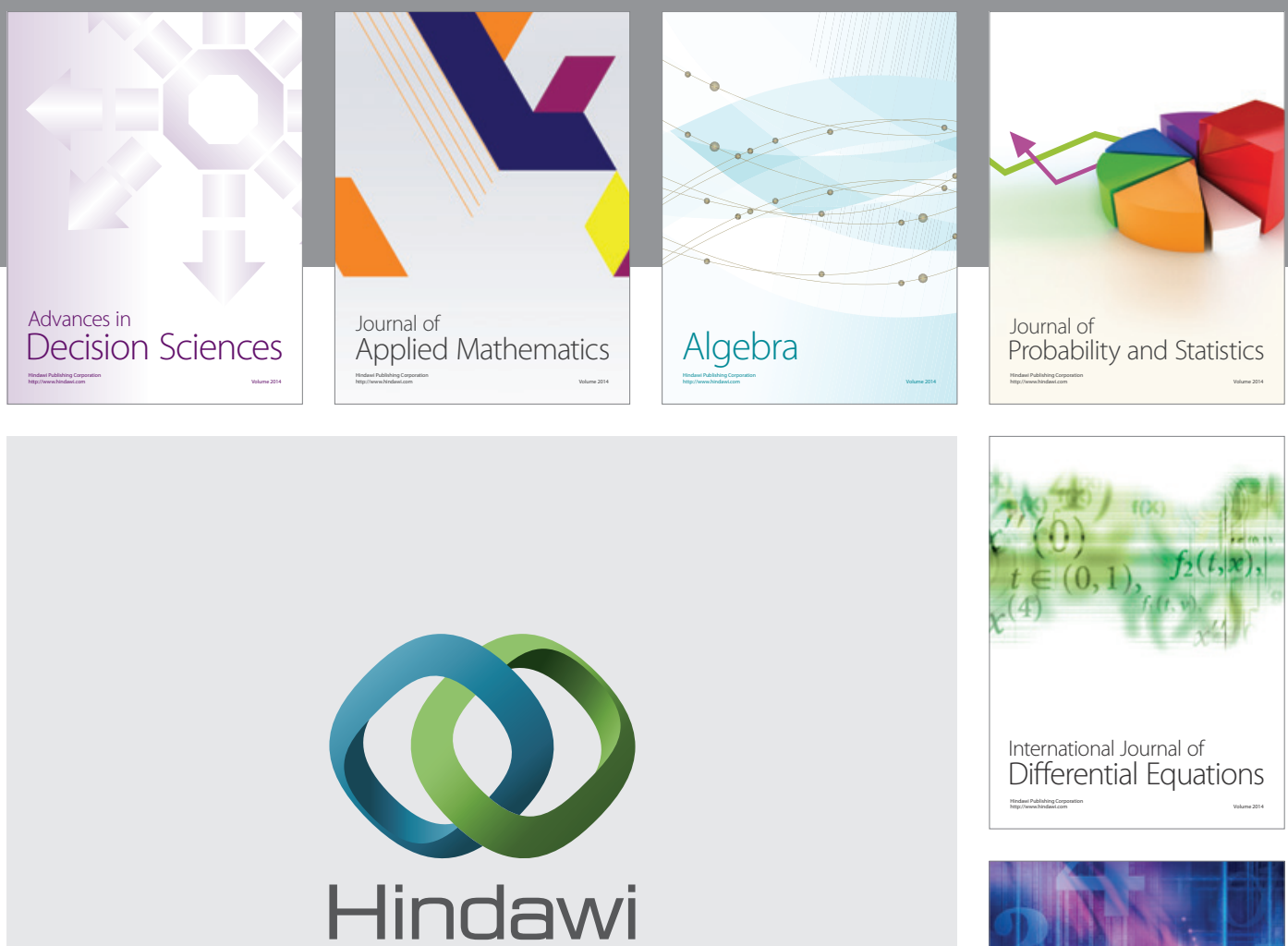

Submit your manuscripts at http://www.hindawi.com
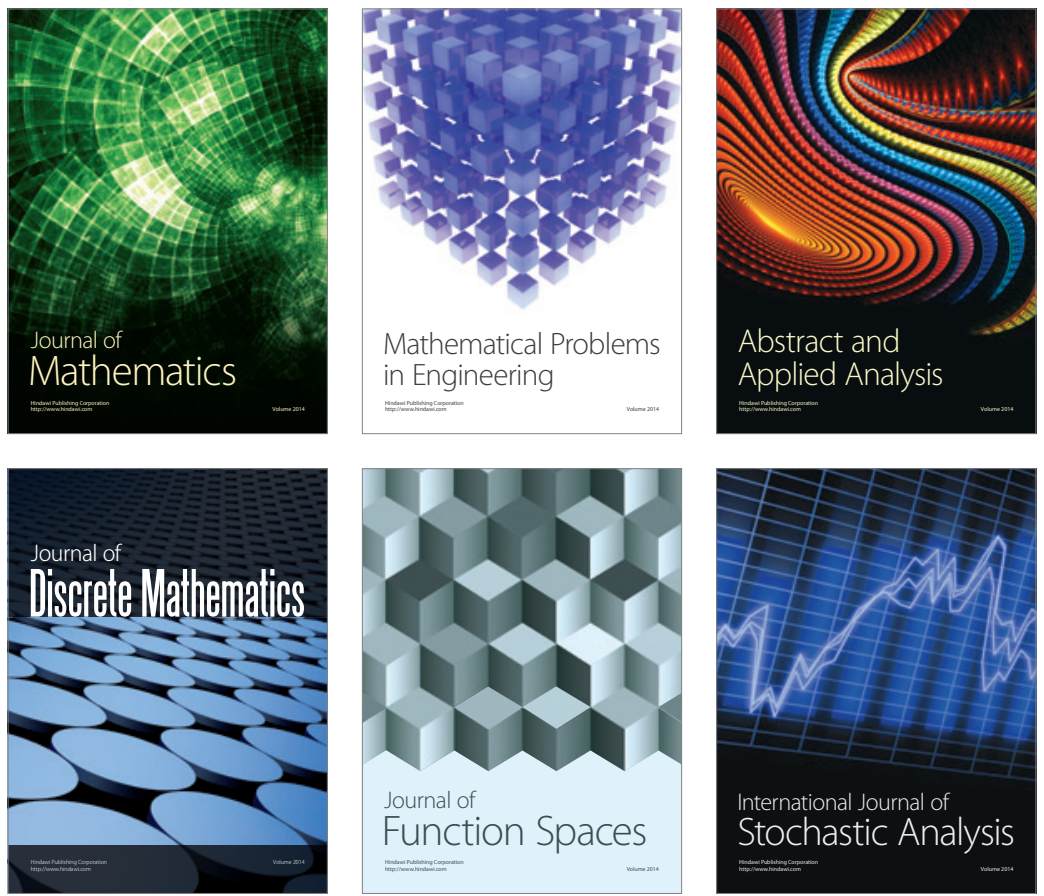

Journal of

Function Spaces

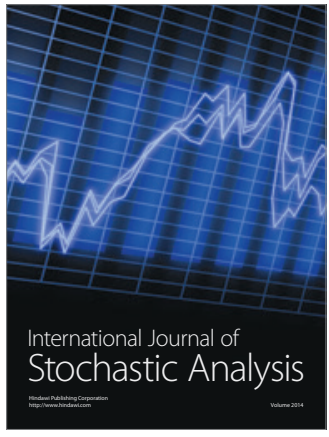

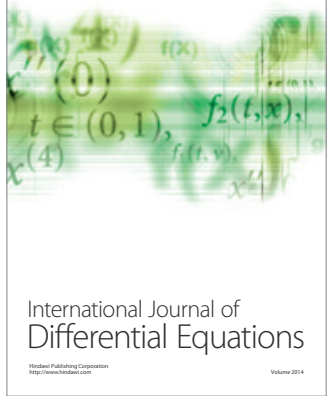
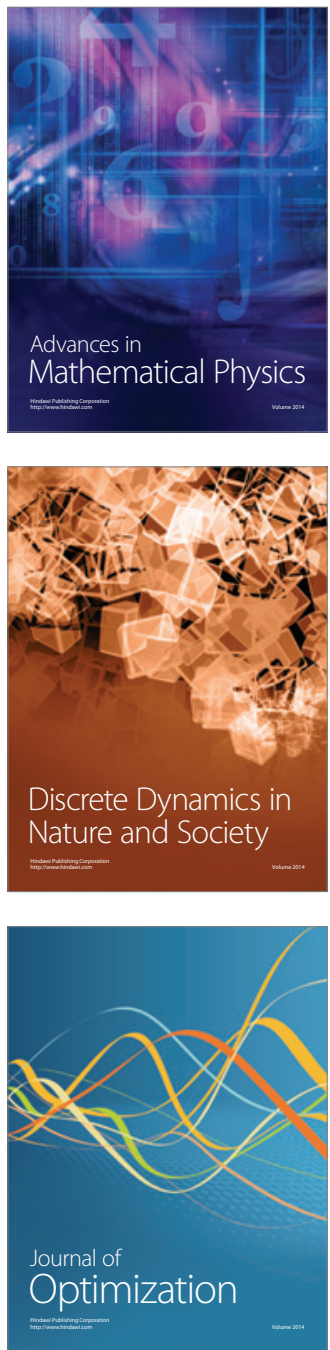